\title{
Determinantes socioeconómicos del estado nutricional en menores de cinco años atendidos en el Hospital Infantil Napoleón Franco Pareja
}

\section{Socioeconomic determinants of nutritional status in children under five attended at the Hospital Infantil Napoleón Franco Pareja}

Deicy Vanessa Moreno-Ruiz ${ }^{1}$, Michelle Marie Picon², Carlos Alberto Marrugo-Arnedo ${ }^{3}$, Verena Marrugo Nelson Rafael Alvis Guzmán ${ }^{1}$

Forma de citar: Moreno-Ruiz DV, Picón MM, Marrugo-Arnedo CA, Verena Marrugo A, Alvis Guzmán NR. Determinantes socioeconómicos del estado nutricional en menores de cinco años atendidos en el Hospital Infantil Napoleón Franco Pareja. Rev Univ Ind Santander Salud. 2017; 49(2): 352-363. DOI: http://dx.doi.org/10.18273/revsal.v49n2-2017008 @c) (1)

\section{RESUMEN}

Objetivo: Determinar los factores sociales y económicos que afectan el estado nutricional de los niños menores de cinco años. Metodología: Se llevó a cabo un estudio de corte transversal con una muestra de 600 menores que asistieron al Hospital Infantil Napoleón Franco Pareja (HINFP) a cuyas madres se les aplicó un instrumento estandarizado para capturar la información socioeconómica y el estado nutricional del niño y de la madre. Se estimaron dos modelos econométricos de Umbral Generalizado, tomando como variable dependientes talla para la edad y peso para la talla y como variables independientes: peso al nacer del menor, número de controles prenatales, nivel de ingresos del hogar, uso de servicios de alcantarillado y acueducto, índice de masa corporal (IMC) de la madre, entre otras. Resultados: De acuerdo al indicador de talla para la edad, el $48.05 \%$ de los niños sufren de desnutrición crónica. El indicador de peso para la talla reflejó que el $22.09 \%$ de los niños tuvieron un déficit en su masa corporal (desnutrición aguda) y un $13.53 \%$ se encontraba en sobrepeso u obesidad. El $76.02 \%$ de las madres con hijos con talla para la edad normal tienen educación media o superior. El 56\% de los niños pertenecientes a hogares con ingresos inferiores al salario mínimo mensual legal vigente tuvieron problemas de desnutrición aguda. Respecto a los determinantes, mayores ingresos reducen la probabilidad de que un niño sufra desnutrición. El tiempo de lactancia materna, la ocupación y el estado nutricional de la madre resultaron ser factores protectores. El incremento de la edad gestacional al nacimiento, aumenta la probabilidad de tener una talla normal. Conclusiones: La presente investigación genera evidencia para revisar la política sanitaria de nutrición infantil. Elevar el nivel de ingresos y promover la lactancia materna mejorarían el estado nutricional de los niños.

Palabras claves: Nutrición infantil, determinantes, indicadores de salud, peso para la estatura, salud pública.

1. Universidad de Cartagena. Bolívar, Colombia

2. University of Miami Miller School of Medicine Miami. Florida, Estados Unidos de América.

3. Centro de Investigación e Innovación en Salud de Coosalud EPS -Cartagena. Bolívar, Colombia.

4. Universidad San Buenaventura. Cartagena, Bolívar, Colombia

Correspondencia: Deicy Vanessa Moreno Ruiz. Dirección: Universidad de Cartagena, Sede Piedra de Bolívar. Avenida del Consulado, Calle 30 48-152. Bloque B - Oficina BS04, Cartagena, Colombia. Correo electrónico: deicyvanessa@hotmail.com. Teléfono: +7 3005251923. 


\section{ABSTRACT}

Objective: To determine the social and economic factors that affect he nutritional status of children under five years of age. Methods: A cross-sectional study carried out with a sample of 600 children were attended the Children's Hospital Napoleón Franco Pareja (HINFP) whose mothers were administered a standardized instrument to capture the socioeconomic information and nutritional status of the child. Two econometric models were estimated using Generalized Threshold, taking as dependent variable-height for age and weight for height and as independent variables: birth weight of the child, number of prenatal controls, level of household income, use of sewerage and aqueduct services, mother's body mass index (BMI), among others. Results: According to the height for age indicator, $48.05 \%$ of children suffer from chronic malnutrition. The indicator weight for height showed that $22.09 \%$ of children have a deficit in their body mass (acute malnutrition) and $13.53 \%$ were overweight or obese. $76.02 \%$ of mothers of children with normal height for age have completed secondary or higher education. $56 \%$ of children in households with incomes below current legal monthly minimum wage have acute malnutrition problems. Regarding the determinants, higher incomes reduce the likelihood that a child would suffer malnutrition. Breastfeeding time, occupation and maternal nutritional status are protective factors. Increasing gestational age at birth increases the likelihood of a normal size. Conclusions: This research generated evidence for health policy review of child nutrition. The raising of incomes and the promotion breastfeeding would improve the nutritional status of children.

Keywords: infant nutrition, determinants, health indicators, weight by height, public health.

\section{INTRODUCCIÓN}

El estado nutricional en los primeros años de vida es decisivo en los resultados futuros de educación, ingresos y salud ${ }^{1-5}$. Debido a su gran importancia, estas relaciones han sido ampliamente estudiadas por investigadores que han asociado indicadores nutricionales (salud) con variables socioeconómicas, lo anterior para dar una mejor explicación del fenómeno de mala nutrición y recomendar políticas públicas que ataquen de forma frontal el problema ${ }^{2,6-8}$.

Para la medición del estado nutricional, la literatura refleja que usualmente se utilizan tres indicadores antropométricos: peso para la talla $(\mathrm{P} / \mathrm{T})$, talla para la edad $(\mathrm{T} / \mathrm{E})$ y peso para la edad $(\mathrm{P} / \mathrm{E})^{9-11}$. El déficit del primero implica disminución en la masa corporal, lo cual hace referencia a desnutrición aguda (DA), el segundo refleja un menor crecimiento lineal en el tiempo, es decir, desnutrición crónica (DC) y el déficit del último se refiere a desnutrición global (DG) ${ }^{2,11}$.

La desnutrición infantil, es uno de los problemas más serios que enfrenta el mundo, ya que no sólo tiene implicaciones sociales sino también económicas, tales como, pérdida en la habilidad cognitiva de los menores, deserción escolar, entre otros aspectos que causan una menor productividad laboral, poniendo en riesgo el crecimiento económico ${ }^{13-17}$. Las implicaciones también se ven reflejadas en el aumento de las tasas de morbilidad y mortalidad infantil. El informe sanitario del 2015 de la Organización Mundial de la Salud (OMS), reportó que la desnutrición es la causa de fondo de un $45 \%$ de las defunciones de menores de 5 años ${ }^{17}$.

Sin embargo, en los últimos años, la Organización de las Naciones Unidas (ONU) por medio del impulso de los Objetivos de Desarrollo Sostenible (ODS) ha buscado mejorar la calidad de vida de las personas, en especial la de la niñez. El segundo objetivo 2030, consiste en poner fin al hambre y asegurar el acceso de todas las personas, a una alimentación sana, nutritiva y suficiente durante todo el año. Así mismo el tercer objetivo busca poner fin a las muertes evitables de recién nacidos y de niños menores de 5 años ${ }^{18}$.

En Colombia, la Encuesta Nacional de Situación Nutricional (ENSIN) 2010, arrojó que el 13.2\% de niños menores de cinco años sufren de desnutrición crónica, porcentaje que está 5 puntos porcentuales por encima de la meta propuesta ( $8 \%$ en el 2015) por la ONU con el fin de cumplir con los $\mathrm{ODS}^{19}$. En tal sentido, reducir la mortalidad infantil, como ODS, implica intervenir en los problemas de desnutrición como factores determinantes de muerte en niños ${ }^{13}$. En ese orden de ideas, el objetivo principal de este estudio es determinar los factores sociales y económicos asociados al estado nutricional de la población infantil en Cartagena, Colombia.

\section{METODOLOGÍA}

Se llevó a cabo un estudio de corte transversal con el objetivo de conocer los factores sociales y económicos que afectan el estado nutricional de niños que asisten al Hospital infantil Napoleón Franco Pareja (HINFP) 
mediante el uso de dos indicadores nutricionales de largo plazo, que capturan la historia nutricional del niño hasta el momento en que es medido $(\mathrm{T} / \mathrm{E}-\mathrm{P} / \mathrm{T})^{20}$. No se tuvo en cuenta el indicador de $\mathrm{P} / \mathrm{E}$, debido a que es más impreciso para estudios poblacionales y puede o no incluir la DA y/o la DC ${ }^{2,11}$. Para medir el estado nutricional de las madres se utilizó el índice de masa corporal (IMC). La OMS también recomienda el uso del IMC para la edad, pero en este estudio no se tuvo en cuenta, se decidió usar las dos medidas antropométricas más frecuentes en la literatura y las que representan mejor el estado nutricional de los niños ${ }^{20}$.

\section{Recolección de datos}

Los datos fueron recolectados por medio de un formulario estandarizado y aplicado a las madres de niños menores de cinco años que asistían a cita pediátrica de rutina en el HINFP durante el segundo semestre de 2015 (Los niños que asisten a este hospital pertenecen al estrato socioeconómico medio y bajo). El formulario preguntaba por características socioeconómicas, demográficas y de acceso a servicios de salud del niño y la madre.

\section{Definición de variables}

Esta investigación utiliza como variables dependientes, dos indicadores antropométricos recomendados por la literatura.

- Puntaje $Z$ - T/E: Es una medida de estatura por edad normalizada a los estándares de la población de referencia de la OMS ${ }^{1}$. El indicador se calcula como la diferencia entre la talla del infante y la talla de referencia de un infante sano de la misma edad y el mismo sexo, dividida por el valor de referencia de la desviación estándar de un niño sano².

\section{$T / E=\frac{\text { Talla Infante Medido-Talla de Referencia }(O M S)}{\text { Desviación Estandar }}$}

- Puntaje $\boldsymbol{Z}-\mathbf{P} / \mathbf{T}$ : Este índice compara el peso de un individuo con el peso esperado para su talla, lo que permite establecer si ha ocurrido una pérdida reciente de peso corporal (DA). Por lo que la ventaja principal es que determina bien al niño adelgazado agudamente de aquel que tiene DC.

$$
P / T=\frac{\text { Peso Infante medido }}{\text { Peso ideal para talla actual (OMS) }}
$$

1. La población de referencia es la que aporta la Organización Mundial de la Salud (OMS), tanto para talla como para peso.

\section{Análisis estadístico}

El presente estudio describe e identifica los factores sociales y económicos que inciden en el estado nutricional de los niños del HINFP. Para la descripción de las variables cualitativas se utilizaron frecuencias absolutas y relativas. Para las variables cuantitativas se utilizaron medidas de tendencia central con sus medidas de dispersión. Tanto la construcción de los indicadores como el análisis de los datos, se realizaron en el paquete estadístico Stata 13.

\section{Modelo econométrico}

Para medir el efecto de las variables socioeconómicas y nutricionales que afectan el estado nutricional de los niños, se empleó un modelo de regresión logística. Dado que la variable dependiente en este modelo es categórica y ordenada ${ }^{2,21}$, se usó el modelo de regresión logística de Umbral generalizado, que pertenece a la familia de modelos de respuesta ordenada, el cual arroja la probabilidad de que un niño pertenezca a una de las categorías a evaluar ${ }^{2,21}$.

Se puede escribir de la siguiente forma:

$$
S=\varnothing\left(X_{1}, X_{h}, W_{h}, X_{c}, \mu\right)
$$

Donde $X_{1}$ representa las características del niño (semanas de gestación, peso al nacer, orden de nacimiento, sexo, lactancia materna, inicio de alimentación complementaria, pertenencia a programas de asistencia nutricional y vacunas), $X_{h}$ es un vector de características del hogar (número de personas que viven en el hogar, uso de servicios de alcantarillado y acueducto, material del piso y paredes), $W_{h}$ representa la riqueza del hogar (estrato, nivel de ingresos), $X_{c}$ un vector que representa las características de la madre (estado civil, IMC, escolaridad, ocupación) y finalmente, $\mu$ representa las variables no observadas y otros efectos. Estas variables están sustentadas en la literatura como posibles factores determinantes del estado nutricional ${ }^{1,4,7,9-12}$.

Para efectos de la presente investigación, se estimaron dos modelos, en el primero se toma como variable explicada el indicador $\mathrm{T} / \mathrm{E}$, que puede tomar las siguientes categorías, talla normal, DC leve, DC moderada y DC severa. El segundo modelo tiene como variable explicada el indicador $\mathrm{P} / \mathrm{T}$, cuyas categorías son, normal, DA y sobrepeso u obesidad. La validación del modelo es estimada a partir del poder predictivo, siendo verificada a través de la prueba Hosmer-

2. Las categorías que se miden en la función de producción de salud son: normal, desnutrición leve, desnutrición moderada, desnutrición severa. 
Lemeshow, que se apoya en un test Chi-cuadrado, que confronta la discrepancia entre lo observado y lo estimado dividiendo los datos en subgrupos.

\section{RESULTADOS}

La Tabla 1 proporciona información acerca del indicador de peso para la talla reflejando que, el
$35.62 \%$ de los niños tienen un estado nutricional que no es normal, distribuidos entre desnutrición aguda y sobrepeso u obesidad, 22,09\% y $13.53 \%$ respectivamente. Por su parte, el indicador de talla para la edad, mostrado en la Tabla 2, evidenció que el $48.05 \%$ de los niños tienen un retraso en el crecimiento lineal y llama la atención que para el $13.87 \%$ de éstos, dicho retraso es severo.

Tabla 1. Características del menor según estado nutricional $(\mathrm{P} / \mathrm{T})$.

\begin{tabular}{|c|c|c|c|c|c|c|}
\hline \multicolumn{7}{|c|}{ Características del menor } \\
\hline & \multicolumn{2}{|c|}{ Normal } & \multicolumn{2}{|c|}{$\mathbf{D A}^{1}$} & \multicolumn{2}{|c|}{ Sobrepeso u obesidad } \\
\hline & Frecuencia & $\%$ & Frecuencia & $\%$ & Frecuencia & $\%$ \\
\hline & 376 & 64.38 & 129 & 22.09 & 79 & 13.53 \\
\hline \multicolumn{7}{|l|}{ Sexo } \\
\hline Femenino & 171 & 45.48 & 62 & 48.06 & 36 & 45.57 \\
\hline Masculino & 205 & 54.52 & 67 & 51.94 & 43 & 54.43 \\
\hline \multicolumn{7}{|l|}{ Afiliación al SGSSS } \\
\hline Subsidiado & 366 & 97.34 & 127 & 98.45 & 77 & 97.47 \\
\hline Otro & 10 & 2.66 & 2 & 1.55 & 2 & 2.53 \\
\hline \multicolumn{7}{|c|}{ Lactancia materna exclusiva } \\
\hline 0 meses & 95 & 25.27 & 33 & 25.58 & 23 & 29.11 \\
\hline$<2$ meses & 36 & 9.57 & 18 & 13.95 & 6 & 7.59 \\
\hline Entre 2 - 3 meses & 64 & 17.02 & 16 & 12.40 & 13 & 16.46 \\
\hline Entre 4 - 6 meses & 127 & 33.78 & 42 & 32.56 & 29 & 36.71 \\
\hline$>6$ meses & 54 & 14.36 & 20 & 15.5 & 8 & 10.13 \\
\hline \multicolumn{7}{|c|}{ Tiempo total de lactancia } \\
\hline 0 meses & 18 & 4.79 & 11 & 8.53 & 5 & 6.33 \\
\hline Entre 1 - 6 meses & 103 & 27.39 & 47 & 36.43 & 31 & 39.24 \\
\hline Entre 7 - 12 meses & 137 & 36.44 & 37 & 28.68 & 31 & 39.24 \\
\hline Entre 13 - 24 meses & 105 & 27.93 & 29 & 22.48 & 11 & 13.92 \\
\hline$>=25$ meses & 13 & 3.46 & 5 & 3.88 & 1 & 1.27 \\
\hline \multicolumn{7}{|c|}{ Inicio de alimentación complementaria } \\
\hline Entre 0 - 3 meses & 152 & 40.75 & 55 & 42.97 & 30 & 38.46 \\
\hline Entre 4 - 6 meses & 78 & 20.91 & 20 & 15.63 & 20 & 25.64 \\
\hline Entre 7 - 12 meses & 124 & 33.24 & 49 & 38.28 & 28 & 35.9 \\
\hline$>=13$ meses & 19 & 5.09 & 4 & 3.13 & 0 & 0 \\
\hline \multicolumn{7}{|l|}{ Orden de nacimiento } \\
\hline Primero & 196 & 52.13 & 60 & 46.51 & 42 & 53.16 \\
\hline Segundo & 99 & 26.33 & 41 & 31.78 & 23 & 29.11 \\
\hline Tercero & 56 & 14.89 & 21 & 16.28 & 10 & 12.66 \\
\hline Cuarto o más & 25 & 6.65 & 7 & 5.43 & 4 & 5.06 \\
\hline \multicolumn{7}{|c|}{ Esquema de vacunación completo } \\
\hline $\mathrm{Si}$ & 341 & 90.69 & 113 & 87.6 & 72 & 91.14 \\
\hline No & 35 & 9.31 & 16 & 12.4 & 7 & 8.86 \\
\hline
\end{tabular}

1 Desnutrición aguda. 
Tabla 2. Características de la madre según estado nutricional $(\mathrm{P} / \mathrm{T})$.

\begin{tabular}{|c|c|c|c|c|c|c|}
\hline \multicolumn{7}{|c|}{ Características de la madre } \\
\hline & \multicolumn{2}{|c|}{ Normal } & \multicolumn{2}{|c|}{$\mathbf{D A}^{2}$} & \multicolumn{2}{|c|}{ Sobrepeso u obesidad } \\
\hline & Frecuencia & $\%$ & Frecuencia & $\%$ & Frecuencia & $\%$ \\
\hline \multicolumn{7}{|l|}{ Escolaridad } \\
\hline Ninguna & 1 & 0.27 & 0 & 0 & 0 & 0 \\
\hline Primaria incompleta & 10 & 2.66 & 1 & 0.78 & 1 & 1.27 \\
\hline Primaria completa & 9 & 2.39 & 3 & 2.33 & 0 & 0 \\
\hline Secundaria incompleta & 80 & 21.28 & 32 & 24.8 & 13 & 16.46 \\
\hline Secundaria completa & 135 & 35.9 & 43 & 33.33 & 34 & 43.04 \\
\hline Técnico o tecnólogo & 115 & 30.59 & 43 & 33.33 & 24 & 30.38 \\
\hline Universitaria & 26 & 6.91 & 7 & 5.43 & 7 & 8.86 \\
\hline \multicolumn{7}{|l|}{ Ocupación } \\
\hline Desempleada & 9 & 2.39 & 4 & 3.1 & 9 & 11.39 \\
\hline Ama de casa & 297 & 78.99 & 98 & 75.97 & 58 & 73.42 \\
\hline Jubilada & 1 & 0.27 & 1 & 0.78 & 0 & 0 \\
\hline Trabajadora independiente & 37 & 9.84 & 15 & 11.63 & 6 & 7.59 \\
\hline Trabajadora asalariada & 32 & 8.51 & 11 & 8.53 & 6 & 7.59 \\
\hline \multicolumn{7}{|l|}{ Estado Civil } \\
\hline Casada & 50 & 13.3 & 19 & 14.73 & 8 & 10.13 \\
\hline Unión libre & 270 & 71.81 & 89 & 68.99 & 54 & 68.35 \\
\hline Soltera & 54 & 14.36 & 21 & 16.28 & 17 & 21.52 \\
\hline Viuda & 2 & 0.53 & 0 & 0 & 0 & 0 \\
\hline \multicolumn{7}{|l|}{ Estado nutricional } \\
\hline Normal & 138 & 47.26 & 52 & 52 & 31 & 47.69 \\
\hline Desnutrición & 11 & 3.77 & 8 & 8 & 3 & 4.62 \\
\hline Sobrepeso & 93 & 31.85 & 30 & 30 & 19 & 29.23 \\
\hline Obesidad & 50 & 17.12 & 10 & 10 & 12 & 18.46 \\
\hline
\end{tabular}

2 Desnutrición aguda.

Al caracterizar los niños de acuerdo a los distintos estados nutricionales, como se puede ver en la Tabla 3, se observa que el $96.24 \%$ viven en hogares de estrato socioeconómico 1 y 2 . El nivel de ingresos de los hogares no es superior a $\$ 600.000$ en el $60 \%$ de los hogares.

Tabla 3. Características de la vivienda según estado nutricional $(\mathrm{P} / \mathrm{T})$.

\begin{tabular}{|c|c|c|c|c|c|c|}
\hline \multicolumn{7}{|c|}{ Características de la vivienda } \\
\hline & \multicolumn{2}{|c|}{ Normal } & \multicolumn{2}{|c|}{$\mathbf{D A}^{3}$} & \multicolumn{2}{|c|}{ Sobrepeso u obesidad } \\
\hline & Frecuencia & $\%$ & Frecuencia & $\%$ & Frecuencia & $\%$ \\
\hline \multicolumn{7}{|l|}{ Estrato } \\
\hline Bajo & 358 & 96.24 & 127 & 98.45 & 76 & 96.21 \\
\hline Medio & 14 & 3.76 & 2 & 1.55 & 3 & 3.8 \\
\hline \multicolumn{7}{|c|}{ Dispone de alcantarillado } \\
\hline $\mathrm{Si}$ & 268 & 71.28 & 102 & 79.07 & 57 & 72.15 \\
\hline No & 108 & 28.72 & 27 & 20.93 & 22 & 27.85 \\
\hline \multicolumn{7}{|c|}{ Dispone de acueducto } \\
\hline $\mathrm{Si}$ & 338 & 89.89 & 114 & 88.37 & 71 & 89.87 \\
\hline No & 38 & 10.11 & 15 & 11.63 & 8 & 10.13 \\
\hline
\end{tabular}




\begin{tabular}{lcccccc}
\hline \multicolumn{3}{c}{ Características de la vivienda } & \multicolumn{3}{c}{ DA $^{3}$} & \multicolumn{3}{c}{ Sobrepeso u obesidad } \\
\hline & \multicolumn{2}{c}{ Normal } & Frecuencia & $\%$ & Frecuencia & $\%$ \\
\hline Material del piso & Frecuencia & $\%$ & & & & \\
Baldosa o cemento & 338 & 89.89 & 120 & 93.02 & 70 & 88.61 \\
Otro & 38 & 10.11 & 9 & 6.98 & 9 & 11.39 \\
Material de las paredes & & & & & & \\
Bloque o ladrillo & 325 & 86.44 & 112 & 86.82 & 72 & 91.14 \\
Otro & 51 & 13.56 & 17 & 13.18 & 7 & 8.86 \\
Nivel de Ingresos & & & & & & 12 \\
Menos de $\$ 300.000$ & 81 & 21.6 & 26 & 20.3 & 12 & 15.19 \\
Entre $\$ 300.000$ y $\$ 600.000$ & 144 & 38.4 & 46 & 35.9 & 26 & 32.9 \\
Entre $\$ 600.001$ y $\$ 900.000$ & 97 & 25.87 & 36 & 28.13 & 30 & 38 \\
Entre $\$ 900.001$ y $\$ 1.200 .000$ & 24 & 6.4 & 12 & 9.40 & 9 & 11.4 \\
Más de $\$ 1.200 .000$ & 29 & 7.73 & 8 & 6.25 & 2 & 2.53 \\
\hline
\end{tabular}

3 Desnutrición aguda.

En lo que respecta a las madres de niños con un adecuado estado nutricional, la edad promedio es de 26 años. El 36\% terminaron sus estudios de bachillerato y aproximadamente el 30.6\% manifestó haber terminado estudios técnicos o tecnológicos y solo un 7\% cuenta con educación superior. En cuanto a la ocupación se encontró que aproximadamente el $78.99 \%$ de las madres son amas de casa, en contraste con las que aportan ingresos al hogar que representan el $18.35 \%$. Por su parte, $85 \%$ de las madres manifestaron tener pareja. A su vez, $47.2 \%$ de las madres tiene un estado nutricional normal de acuerdo a su IMC. Llama la atención el alto porcentaje de madres en sobrepeso u obesidad (48.97 \%). Estos datos se pueden ver en la Tabla 2.

El 95.2\% de los niños sanos tuvo durante su gestación más de cuatro visitas a control prenatal y en promedio nacieron con 38 semanas de gestación, pesando 3,133 gramos. El $60.37 \%$ de los niños recibieron lactancia materna exclusiva entre uno y seis meses. Así mismo, el porcentaje más alto de niños con adecuado estado nutricional (36.44\%) que recibieron algún tipo de lactancia materna, la recibieron durante 7-12 meses.

El $91 \%$ de los niños con adecuada talla para la edad, tienen su esquema de vacunación completo. Así mismo, las madres de estos niños se caracterizan por ser en su mayoría amas de casa $(75,2 \%)$ y vivir en unión libre $(68,1 \%)$. En cuanto a las características de la vivienda de los niños con adecuada talla para la edad se encontró que, no existen marcadas diferencias, respecto a los demás estados nutricionales. Lo que podría explicarse por el hecho de que el $96.51 \%$ de los niños viven en los estratos socioeconómicos 1 y 2 , lo que evidencia una homogeneidad entre estos niños. Estos resultados se pueden ver en las Tablas 4, 5 y $\mathbf{6}$ respectivamente.

Tabla 4. Características del menor según estado nutricional (T/E).

\begin{tabular}{|c|c|c|c|c|c|c|c|c|}
\hline \multicolumn{9}{|c|}{ Características del niño } \\
\hline & \multicolumn{2}{|c|}{ Normal } & \multicolumn{2}{|c|}{ DC $^{4}$ leve } & \multicolumn{2}{|c|}{ DC moderada } & \multicolumn{2}{|c|}{ DC severa } \\
\hline & Frecuencia & $\%$ & Frecuencia & $\%$ & Frecuencia & $\%$ & Frecuencia & $\%$ \\
\hline & 307 & 51.95 & 143 & 24.2 & 59 & 9.98 & 82 & 13.87 \\
\hline \multicolumn{9}{|l|}{ Sexo } \\
\hline Femenino & 147 & 47.88 & 61 & 42.66 & 27 & 45.76 & 38 & 46.34 \\
\hline Masculino & 160 & 52.12 & 82 & 57.34 & 32 & 54.24 & 44 & 53.66 \\
\hline \multicolumn{9}{|c|}{ Afiliación al SGSSS } \\
\hline Subsidiado & 298 & 97.07 & 139 & 97.2 & 58 & 98.31 & 82 & 100 \\
\hline Otro & 9 & 2.93 & 4 & 2.8 & 1 & 1.69 & 0 & 0 \\
\hline
\end{tabular}


Determinantes socioeconómicos del estado nutricional en menores de cinco años atendidos en el Hospital Infantil Napoleón Franco Pareja

\begin{tabular}{|c|c|c|c|c|c|c|c|c|}
\hline \multicolumn{9}{|c|}{ Características del niño } \\
\hline & \multicolumn{2}{|c|}{ Normal } & \multicolumn{2}{|c|}{ DC $^{4}$ leve } & \multicolumn{2}{|c|}{ DC moderada } & \multicolumn{2}{|c|}{ DC severa } \\
\hline & Frecuencia & $\%$ & Frecuencia & $\%$ & Frecuencia & $\%$ & Frecuencia & $\%$ \\
\hline \multicolumn{9}{|c|}{ Lactancia materna exclusiva } \\
\hline 0 meses & 77 & 25.08 & 37 & 25.87 & 14 & 23.73 & 28 & 34.15 \\
\hline$<2$ meses & 33 & 10.75 & 19 & 13.29 & 3 & 5.08 & 6 & 7.32 \\
\hline Entre 2 - 3 meses & 56 & 18.24 & 19 & 13.29 & 7 & 11.86 & 12 & 14.63 \\
\hline Entre 4 - 6 meses & 97 & 31.6 & 51 & 35.66 & 24 & 40.86 & 26 & 31.71 \\
\hline$>6$ meses & 44 & 14.33 & 17 & 11.89 & 11 & 18.64 & 10 & 12.2 \\
\hline \multicolumn{9}{|c|}{ Tiempo total de lactancia } \\
\hline 0 meses & 17 & 5.54 & 8 & 5.59 & 4 & 6.78 & 7 & 8.54 \\
\hline Entre 1 - 6 meses & 91 & 29.46 & 48 & 33.57 & 17 & 28.81 & 27 & 32.93 \\
\hline Entre 7 - 12 meses & 115 & 37.46 & 43 & 30.07 & 23 & 38.98 & 27 & 32.93 \\
\hline Entre $13-24$ meses & 73 & 23.78 & 38 & 26.57 & 14 & 23.73 & 20 & 24.39 \\
\hline$>=25$ meses & 11 & 3.58 & 6 & 4.2 & 1 & 1.69 & 1 & 1.22 \\
\hline \multicolumn{9}{|c|}{ Inicio de alimentación complementaria } \\
\hline Entre 0 - 3 meses & 128 & 42.24 & 60 & 42.25 & 18 & 30.51 & 37 & 45.12 \\
\hline Entre 4 - 6 meses & 66 & 21.78 & 22 & 15.49 & 14 & 23.73 & 17 & 20.73 \\
\hline Entre 7 - 12 meses & 99 & 32.67 & 53 & 37.32 & 23 & 38.98 & 26 & 31.71 \\
\hline$>=13$ meses & 10 & 3.3 & 7 & 4.93 & 4 & 6.78 & 2 & 2.44 \\
\hline \multicolumn{9}{|l|}{ Orden de nacimiento } \\
\hline Primero & 154 & 50.16 & 73 & 51.05 & 31 & 52.54 & 44 & 53.66 \\
\hline Segundo & 87 & 28.34 & 45 & 31.47 & 12 & 20.34 & 21 & 25.61 \\
\hline Tercero & 51 & 16.61 & 16 & 11.19 & 11 & 18.64 & 10 & 12.2 \\
\hline Cuarto o más & 15 & 4.89 & 9 & 6.29 & 5 & 8.47 & 7 & 8.54 \\
\hline \multicolumn{9}{|c|}{ Esquema de vacunación completo } \\
\hline $\mathrm{Si}$ & 280 & 91.21 & 128 & 89.51 & 52 & 88.14 & 73 & 89.02 \\
\hline No & 27 & 8.79 & 15 & 10.49 & 7 & 11.86 & 9 & 10.98 \\
\hline
\end{tabular}

4 Desnutrición crónica.

Tabla 5. Características de la madre según estado nutricional (T/E).

\begin{tabular}{|c|c|c|c|c|c|c|c|c|}
\hline \multicolumn{9}{|c|}{ Características de la madre } \\
\hline & \multicolumn{2}{|c|}{ Normal } & \multicolumn{2}{|c|}{ DC $^{5}$ leve } & \multicolumn{2}{|c|}{ DC moderada } & \multicolumn{2}{|c|}{ DC severa } \\
\hline & Frecuencia & $\%$ & Frecuencia & $\%$ & Frecuencia & $\%$ & Frecuencia & $\%$ \\
\hline \multicolumn{9}{|l|}{ Escolaridad } \\
\hline Ninguna & 1 & 0.33 & 0 & 0 & 0 & 0 & 0 & 0 \\
\hline Primaria incompleta & 3 & 0.98 & 8 & 5.59 & 0 & 0 & 1 & 1.22 \\
\hline Primaria completa & 6 & 1.95 & 4 & 2.8 & 1 & 1.69 & 1 & 1.22 \\
\hline Secundaria incompleta & 63 & 20.52 & 27 & 18.88 & 18 & 30.51 & 18 & 21.95 \\
\hline Secundaria completa & 109 & 35.5 & 51 & 35.66 & 17 & 28.81 & 40 & 48.78 \\
\hline Técnico o tecnólogo & 103 & 33.55 & 42 & 29.37 & 19 & 32.2 & 18 & 21.95 \\
\hline Universitaria & 22 & 7.17 & 11 & 7.69 & 4 & 6.78 & 4 & 4.88 \\
\hline \multicolumn{9}{|l|}{ Ocupación } \\
\hline Desempleada & 11 & 3.58 & 3 & 2.1 & 2 & 3.39 & 7 & 8.54 \\
\hline Ama de casa & 231 & 75.24 & 118 & 82.52 & 47 & 79.66 & 63 & 76.83 \\
\hline
\end{tabular}


Características de la madre

\begin{tabular}{lcccccccc}
\hline & \multicolumn{2}{c}{ Normal } & \multicolumn{2}{c}{ DC $^{5}$ leve } & DC moderada & \multicolumn{2}{c}{ DC severa } \\
\hline & Frecuencia & $\%$ & Frecuencia & $\%$ & Frecuencia & $\%$ & Frecuencia & $\%$ \\
\hline Ocupación & 2 & 0.65 & 0 & 0 & 0 & 0 & 0 & 0 \\
Jubilada & 32 & 10.42 & 14 & 9.79 & 6 & 10.17 & 6 & 7.32 \\
Trabajadora independiente & 31 & 10.1 & 8 & 5.59 & 4 & 6.78 & 6 & 7.32 \\
Trabajadora asalariada & & & & & & & & \\
Estado Civil & 47 & 15.31 & 22 & 15.38 & 3 & 5.08 & 6 & 7.32 \\
Casada & 209 & 68.08 & 102 & 71.33 & 49 & 83.05 & 59 & 71.95 \\
Unión libre & 50 & 16.29 & 18 & 12.59 & 7 & 11.86 & 17 & 20.73 \\
Soltera & 1 & 0.33 & 1 & 0.7 & 0 & 0 & 0 & 0 \\
Viuda & 124 & 52.77 & 49 & 41.88 & 20 & 42.55 & 32 & 50 \\
Estado nutricional & 9 & 3.83 & 4 & 3.42 & 5 & 10.64 & 4 & 6.25 \\
Normal & 69 & 29.36 & 40 & 34.91 & 15 & 31.91 & 19 & 29.69 \\
Desnutrición & 33 & 14.04 & 24 & 20.51 & 7 & 14.89 & 9 & 14.06 \\
Sobrepeso & & & & & & & & \\
Obesidad & & & & & & & & \\
\hline
\end{tabular}

5 Desnutrición crónica.

Tabla 6. Características de la vivienda según estado nutricional (T/E).

\begin{tabular}{lcccccccc}
\hline \multicolumn{7}{c}{ Características de la vivienda } \\
\hline & \multicolumn{2}{c}{ Normal } & \multicolumn{2}{c}{ DC $^{6}$ leve } & DC moderada & \multicolumn{2}{c}{ DC severa } \\
\cline { 2 - 9 } & Frecuencia & $\%$ & Frecuencia & $\%$ & Frecuencia & $\%$ & Frecuencia & $\%$ \\
\hline Estrato & & & & & & & & \\
Bajo & 295 & 96.41 & 135 & 95.74 & 58 & 98.31 & 79 & 97.53 \\
Medio & 11 & 3.59 & 6 & 4.26 & 1 & 1.69 & 2 & 2.47 \\
Dispone de alcantarillado & & & & & & & & \\
Si & 242 & 78.83 & 99 & 69.23 & 42 & 71.19 & 50 & 60.98 \\
No & 65 & 21.17 & 44 & 30.77 & 17 & 28.81 & 32 & 39.02 \\
Dispone de acueducto & & & & & & & & \\
Si & 283 & 92.18 & 127 & 88.81 & 51 & 86.44 & 69 & 84.15 \\
No & 24 & 7.82 & 16 & 11.19 & 8 & 13.56 & 13 & 15.85 \\
Material del piso & & & & & & & & \\
Baldosa o cemento & 284 & 92.51 & 127 & 88.81 & 53 & 89.83 & 71 & 86.59 \\
Otro & 23 & 7.49 & 16 & 11.19 & 6 & 10.17 & 11 & 13.41 \\
Material de las paredes & & & & & & & & \\
Bloque o ladrillo & 274 & 89.25 & 120 & 83.92 & 52 & 88.14 & 70 & 85.37 \\
Otro & 33 & 10.75 & 23 & 16.08 & 7 & 11.86 & 12 & 14.63 \\
Nivel de Ingresos & & & & & & & & \\
Menos de $\$ 300.000$ & 63 & 20.59 & 27 & 18.88 & 11 & 18.64 & 121 & 20.54 \\
Entre $\$ 300.000$ y $\$ 600.000$ & 105 & 34.31 & 63 & 44.06 & 22 & 37.29 & 218 & 37.01 \\
Entre $\$ 600.001$ y $\$ 900.000$ & 83 & 27.12 & 36 & 25.17 & 22 & 37.29 & 164 & 27.84 \\
Entre $\$ 900.001$ y $\$ 1.200 .000$ & 27 & 8.82 & 10 & 6.99 & 1 & 1.69 & 46 & 7.81 \\
Más de $\$ 1.200 .000$ & 28 & 9.15 & 7 & 4.9 & 3 & 5.08 & 40 & 6.79 \\
\hline Des & & & & & & &
\end{tabular}

6 Desnutrición crónica. 
Los efectos marginales del modelo logístico para el indicador T/E mostrados en la Tabla 7, evidencian que la probabilidad de tener DC moderada es menor con un mayor nivel de ingresos. Esto se confirma con el signo positivo del coeficiente de talla normal $\left(0.11 ; \mathrm{EE}^{3}: 0.09\right)$, explicando que niveles de ingreso superiores aumentan la probabilidad de tener una talla normal con respecto a los hogares cuyo nivel de ingreso es menor a $\$ 300.000$.

Tabla 7. Efectos marginales promedios del modelo de determinantes del estado nutricional dado el indicador T/E.

\begin{tabular}{|c|c|c|c|c|}
\hline \multirow{2}{*}{ Variables } & \multicolumn{4}{|c|}{ Estado nutricional } \\
\hline & $\begin{array}{l}\text { Normal } \\
\beta(\mathbf{E E})^{7}\end{array}$ & $\begin{array}{c}\text { DC Leve } \\
\beta(\mathrm{EE})\end{array}$ & $\begin{array}{c}\text { DC Moderada } \\
\beta(\text { EE) }\end{array}$ & $\begin{array}{c}\text { DC Severa } \\
\beta(\mathbf{E E})\end{array}$ \\
\hline \multicolumn{5}{|l|}{ Nivel de ingresos del hogar } \\
\hline Entre $\$ 300000$ y $\$ 600000$ & $-.071(0.573)$ & $.073(0.048)$ & $.007(0.039)$ & $-.009(0.044)$ \\
\hline Entre $\$ 600001$ y $\$ 900000$ & $-.044(0.606)$ & $-.002(0.054)$ & $.023(0.043)$ & $.024(0.048)$ \\
\hline Más de $\$ 900001$ & $.112(0.906)$ & $.023(0.081)$ & $\begin{array}{l}-.762 * * * \\
(0.0294)\end{array}$ & $.626(0.265)$ \\
\hline Semanas de gestación & $-.003(0.010)$ & $.001(0.009)$ & $-.003(0.008)$ & $.005(0.009)$ \\
\hline Peso al nacer & $.000 * * *(0.000)$ & $-.000 *(0.000)$ & $.000(0.000)$ & $-.001 * * *(0.000)$ \\
\hline \multicolumn{5}{|l|}{ Estado civil de la madre } \\
\hline Soltera & $.001(0.063)$ & $.017(0.056)$ & $.071 *(0.422)$ & $-.090(0.057)$ \\
\hline \multicolumn{5}{|l|}{ Ocupación de la madre } \\
\hline Independiente & $-.005(0.075)$ & $.030(0.067)$ & $-.022(0.059)$ & $-.002(0.070)$ \\
\hline Desempleada & $.0764(0.117)$ & $-.116(0.089)$ & $-.074(0.096)$ & $.115(0.116)$ \\
\hline Asalariada & $.145^{* *}(0.076)$ & $-.087(0.059)$ & $-.017(0.0507)$ & $-.040(0.052)$ \\
\hline Jubilada & $.460 * * *(0.022)$ & $-.460 * * *(0.022)$ & 0 & 0 \\
\hline \multicolumn{5}{|l|}{ Tiempo total de lactancia materna } \\
\hline Entre 1- 6 & $-.046(0.103)$ & $.090(0.089)$ & $-.040(0.069)$ & $-.003(0.073)$ \\
\hline Entre 7- 12 & $.099(0.105)$ & $-.031(0.093)$ & $-.046(0.071)$ & $-.022(0.073)$ \\
\hline Entre $13-24$ & $.043(0.109)$ & $.004(0.094)$ & $-.046(0.063)$ & $-.002(0.071)$ \\
\hline$>=25$ & $.096(0.150)$ & $-.000(0.139)$ & $-.206(0.249)$ & $.111(0.281)$ \\
\hline \multicolumn{5}{|c|}{ Inicio de alimentación complementaria } \\
\hline Entre $4-6$ & $-.033(0.062)$ & $-.028(0.058)$ & $.035(0.049)$ & $.026(0.055)$ \\
\hline Entre $7-12$ & $-.155 * * *(0.058)$ & $.047(0.054)$ & $.100 * *(0.050)$ & $.007(0.053)$ \\
\hline$>=13$ & $-.204 *(0.124)$ & $.093(0.124)$ & $.239 *(0.130)$ & $-.129 * * *(0.037)$ \\
\hline Edad del niño & $-.000(0.001)$ & $.003 * *(0.001)$ & $-.001(0.001)$ & $-.001(0.001)$ \\
\hline \multicolumn{5}{|l|}{ Estado nutricional de la madre } \\
\hline Desnutrición & $-.121(0.113)$ & $.012(0.094)$ & $.073(0.979)$ & $.036(0.096)$ \\
\hline Sobrepeso & $-.100 * *(0.052)$ & $.067(0.047)$ & $.072 *(0.044)$ & $-.039(0.046)$ \\
\hline Obesidad & $-.104(0.069)$ & $.113 *(0.067)$ & $.022(0.045)$ & $-.031(0.05)$ \\
\hline
\end{tabular}

$7 \beta$ : betas y EE: Error estándar.

DC: Desnutrición crónica. $* * * \mathrm{P}<0.01$. $* * \mathrm{P}<0.05 . * \mathrm{P}<0.10$ 
Respecto a la variable peso al nacer, observamos que a medida que incrementa el peso al nacer aumenta la probabilidad (0.0001; EE: 0.000: $p<0,01)^{4}$ de que el niño tenga una talla adecuada para su edad, de igual forma, a medida que aumente su peso al nacer disminuye la probabilidad (-0.01; EE: $0.000 ; p<0,01)$ de padecer DC severa. Para el caso de la ocupación de la madre, se encontró que la probabilidad (0.145; EE: 0.076; $\mathrm{p}<0,05)$ de que los niños tengan un buen estado nutricional aumenta en aquellos cuyas madres tienen un trabajo asalariado. Así mismo, el hecho de que una madre se encuentre en sobrepeso reduce la probabilidad $(-0.10$; EE: $0.05 ; \mathrm{p}<0,05)$ de que un niño tenga un estado nutricional normal.
Por su parte, en la Tabla 8 se muestran los efectos marginales del modelo logístico para el indicador $\mathrm{P} / \mathrm{T}$, los resultados confirman que el peso al nacer es un factor protector, debido a que éste aumenta la probabilidad $(0.001$; EE: $0.000 ; p<0,01)$ de tener un estado nutricional normal, es decir, reduce la probabilidad de tener un déficit en la masa corporal del niño. El tiempo de lactancia materna entre 13 y 24 meses, aumenta la probabilidad (0.25; EE: 0.077; $\mathrm{p}<0,01)$ de que el niño tenga un estado nutricional normal, y a su vez reduce la probabilidad (-0.21; EE: $0.070 ; p<0,01)$ de que sufra de desnutrición aguda.

Tabla 8. Efectos marginales promedios del modelo de determinantes del estado nutricional dado el indicador $\mathrm{P} / \mathrm{T}$.

\begin{tabular}{|c|c|c|c|}
\hline \multirow[b]{2}{*}{ Variables } & \multicolumn{3}{|c|}{ Estado nutricional } \\
\hline & $\begin{array}{l}\text { Normal } \\
\beta(E \mathbf{E})\end{array}$ & $\begin{array}{c}\text { Desnutrición aguda } \\
\beta(E \mathbf{E})\end{array}$ & $\begin{array}{l}\text { Sobrepeso u obesidad } \\
\beta(E E) \times x\end{array}$ \\
\hline \multicolumn{4}{|l|}{ Nivel de ingresos del hogar } \\
\hline Entre $\$ 300000$ y $\$ 600000$ & $-.012(0.054)$ & $-.02(0.047)$ & $.035(0.027)$ \\
\hline Entre $\$ 600001$ y $\$ 900000$ & $-.071(0.058)$ & $-.011(0.535)$ & $.082 *(0.042)$ \\
\hline Más de $\$ 900000$ & $-.160 *(0.095)$ & $.091(0.081)$ & $.0690279(0.061)$ \\
\hline Semanas de gestación & $-.174 *(0.009)$ & $.013(0.008)$ & $.0036259(0.004)$ \\
\hline Peso al nacer & $.001 * * *(0.000)$ & $-.021 * * *(0.000)$ & $.001(0.000)$ \\
\hline \multicolumn{4}{|l|}{ Estado civil de la madre } \\
\hline Soltera & $.085(0.061)$ & $-.043(0.053)$ & $-.041(0.035)$ \\
\hline \multicolumn{4}{|l|}{ Ocupación de la madre } \\
\hline Independiente & $-.026(0.079)$ & $.062(0.070)$ & $-.035 *(0.021)$ \\
\hline Desempleada & $-.261 * *(0.126)$ & $.127(0.096)$ & $.133(0.085)$ \\
\hline Asalariada & $-.014(0.076)$ & $.005(0.066)$ & $.008(0.039)$ \\
\hline Jubilada & $-.195(0.282)$ & $.269(0.282)$ & $-.074 * * *(0.010)$ \\
\hline \multicolumn{4}{|l|}{ Tiempo total de lactancia materna } \\
\hline Entre $1-6$ & $.122(0.087)$ & $-.105(0.079)$ & $-.017(0.034)$ \\
\hline Entre 7- 12 & $.259 * * *(0.084)$ & $-.237 * * *(0.075)$ & $-.021(0.039)$ \\
\hline Entre 13- 24 & $.250 * * *(0.077)$ & $-.213 * * *(0.070)$ & $-.037(0.036)$ \\
\hline$>=25$ & $.169 *(0.096)$ & $-.131(0.091)$ & $-.037(0.043)$ \\
\hline \multicolumn{4}{|l|}{$\begin{array}{l}\text { Inicio de la alimentación } \\
\text { complementaria }\end{array}$} \\
\hline Entre $4-6$ & $-.083(0.064)$ & $.037(0.053)$ & $.046(0.035)$ \\
\hline Entre 7 - 12 & $-.185 * * *(0.063)$ & $.153^{* * *}(0.055)$ & $.031(0.036)$ \\
\hline$>=13$ & $.027(0.136)$ & $.085(0.134)$ & $-.113 * * *(0.015)$ \\
\hline Edad del niño & $.004 * *(0.001)$ & $-.002 *(0.014)$ & $-.001(0.000)$ \\
\hline \multicolumn{4}{|l|}{ Estado nutricional de la madre } \\
\hline Desnutrición & $-.218 * *(0.104)$ & $.203 * *(0.101)$ & $.014(0.054)$ \\
\hline Sobrepeso & $-.016(0.516)$ & $.024(0.045)$ & $-.007(0.023)$ \\
\hline Obesidad & $.058(0.595)$ & $-.096 *(0.054)$ & $.038(0.037)$ \\
\hline
\end{tabular}

$* * * \mathrm{P}<0.01 . * * \mathrm{P}<0.05$. $* \mathrm{P}<0.10$

4. Hace referencia al nivel de significancia 
Respecto a edad en meses del niño, se evidencia que los niños de mayores edades son menos vulnerables a la desnutrición aguda $(-0.002$; EE: 0.001: $\mathrm{p}<0,10)$. Finalmente, la desnutrición en las madres reduce la probabilidad $(-0.218$; EE: $0.104 \mathrm{p}<0,05)$, de que un niño tenga un peso adecuado a su talla.

\section{DISCUSIÓN}

En este estudio se presenta evidencia que relaciona el estado nutricional de los niños (puntaje $Z$ de T/E y $\mathrm{P} / \mathrm{T}$ ) con variables socioeconómicas de la población atendida en el HINFP. Los resultados muestran, por un lado, que el ingreso es un factor determinante del estado nutricional de los niños y, por lo tanto, del estado de salud. Por su parte, la edad de los niños en meses, tuvo un comportamiento protector, es decir, entre mayor sea el niño es menos probable que posea mala nutrición.

En cuanto a los resultados que relacionan riqueza con el estado nutricional del menor, se evidencia una relación similar con estudios previos ${ }^{1,12,22,23}$. No obstante, con respecto a la variable número de hijos, que es una proxy de menor cantidad de recursos al interior del hogar, se encontró que los resultados de la presente investigación no son acordes con otros estudios realizados en Colombia, Perú y Ecuador, estudios en los que la variable número de hijos si generó diferencias significativas en la $\mathrm{DC}^{4,11,24}$. Probablemente los resultados de esta investigación no son acordes, puesto que, la muestra de nuestro estudio se centra en población de estratos bajo y medio, y al concentrarse el ingreso en el lado izquierdo de la curva de distribución, la diferencia entre el número de hijos se convierte estadísticamente no significativa.

Respecto a la edad, el estado nutricional y la escolaridad de la madre, los resultados fueron similares a otros estudios, se evidencia que a medida que incrementan, mejora el estado nutricional de los niños ${ }^{1,11,24-28}$ wasting and overweight among infants of urban and rural areas in the Ecuadorian highlands. DESIGN Cross-sectional study. SETTING Nabon (rural. De igual modo, existe una fuerte asociación entre los controles prenatales y el estado nutricional de los niños, como lo muestran estudios realizados en Argentina y la India, donde un menor número de controles aumenta la probabilidad de mal estado nutricional ${ }^{23,29}$.

Respecto a la afiliación al SGSSS, la asociación no fue estadísticamente significativa, esto difiere con los resultados de Tovar, et al. $(2005)^{30}$ en los que la afiliación al SGSSS jugó un papel importante en el estado nutricional de los niños e influyó en la demanda de servicios médicos y en el número de hijos al interior de un hogar. Esta diferencia probablemente se debe a que los niveles de aseguramiento en salud en 2005 eran sensiblemente inferiores al alcanzado hoy día. Por último, la alimentación complementaria se convierte en un factor protector, disminuyendo el riesgo de mala nutrición.

El presente estudio presenta limitaciones, una de ellas está relacionada con el diseño del estudio, al ser una encuesta de corte transversal no está clara la temporalidad de algunas variables, por lo que sólo podemos decir que hay asociaciones significativas y no causalidad. Otra limitación es que la población de estudio solo incluye niños de estrato socioeconómico bajo, lo que genera que no puedan encontrarse diferencias significativas en algunas variables, como la afiliación al sistema de salud o el número de hijos. Sin embargo, el presente estudio presenta fortalezas importantes, el posible sesgo de información de datos se minimiza por la exhaustiva recolección de datos en una encuesta local.

En conclusión, se evidenciaron que las diferencias en la talla para la edad de los niños son significativas según el nivel de ingresos del hogar, es decir, a mayor cantidad de ingresos menor es la probabilidad de mala nutrición. Elevar el ingreso de los hogares, así como la escolaridad de la madre podría jugar un papel fundamental y mejorar el estado nutricional de los niños. En Colombia, las políticas públicas para incrementar los ingresos de los hogares de estrato socioeconómico bajos han estado enfocadas por la vía de los subsidios, entregando recursos de acuerdo al número de hijos. Pero estas medidas han generado que las familias tengan un incentivo a tener más hijos, lo que acentúa los niveles de pobreza. En este sentido, consideramos que las políticas públicas deben enfocarse en incrementar el nivel de educación de la sociedad, ya que por esta vía no solo se logra que las madres estén más informadas respecto al cuidado de sus hijos, sino que también constituye una fuente para mejorar los ingresos de los padres de familia.

\section{CONFLICTO DE INTERESES}

Los autores declaran no tener conflictos de intereses.

\section{AGRADECIMIENTOS}

Alprogramade Jóvenes Investigadores delDepartamento Administrativo de Ciencia, Tecnología e Innovación COLCIENCIAS por la financiación de la investigación, a la Universidad de Cartagena y al Hospital Infantil Napoleón Franco pareja, por permitir la recolección de los datos. 


\section{REFERENCIAS}

1. Delpeuch F, Traissac P, Martin-Prével Y, Massamba J, Maire B. Economic crisis and malnutrition: socioeconomic determinants of anthropometric status of preschool children and their mothers in an African urban area. Public Health Nutr. 2000; 3(01).

2. Marrugo-Arnedo C, Moreno-Ruiz D, Castro-Ávila R, Paternina-Caicedo Á, Marrugo-Arnedo V, AlvisGuzmán N. Sociodemographic Determinants of child nutrition in Colombia. Salud Uninorte. 2015; 31(3): 446-457.

3. Larrea C, Kawachi I. Does economic inequality affect child malnutrition? The case of Ecuador. Soc Sci Med. 2005; 60(1): 165-178.

4. Gaviria AU, del Mar Palau MM. Nutrición y salud infantil en Colombia: determinantes y alternativas de política. Coyunt Económica. 2006; XXXVI(2).

5. Pelletier DL, Frongillo EA, Schroeder DG, Habicht JP. The effects of malnutrition on child mortality in developing countries. Bull World Health Organ. 1995; 73(4): 443-448.

6. Linnemayr S, Alderman H, Ka A. Determinants of malnutrition in Senegal: Individual, household, community variables, and their interaction. Econ Hum Biol. 2008; 6(2): 252-263.

7. Acosta Karina. La desnutrición en los primeros años de vida: Un análisis regional para Colombia. Vol. 160, Documentos de Trabajo sobre Economía Regional. 2012.

8. Ruel M, Hoddinott J. Investing in early childhood nutrition: Policy briefs. International Food Policy Research Institute (IFPRI); 2008.

9. Jesmin A, Yamamoto SS, Malik AA, Haque MA. Prevalence and Determinants of Chronic Malnutrition among Preschool Children: A Crosssectional Study in Dhaka City, Bangladesh. J Heal Popul Nutr. 2011; 29(5): 494-499.

10. Fakir AMS, Khan MWR. Determinants of malnutrition among urban slum children in Bangladesh. Health Econ Rev. 2015; 5(1): 22.

11. Sobrino M, Gutiérrez C, Cunha AJ, Dávila M, Alarcón J. Desnutrición infantil en menores de cinco años en Perú: tendencias y factores determinantes. Rev Panam Salud Publica. 2014; 35(2).

12. Paraje G. Desnutrición crónica infantil y desigualdad socioeconómica en América Latina y el Caribe. Rev Cepal. 2009; 99.

13. Fondo de las Naciones Unidas para la Infancia (UNICEF). La Desnutrición Infantil: Causas, consecuencias y estrategias para su prevención y tratamiento. 2011.

14. Almond D, Currie J. Human Capital Development Before Age Five. Cambridge, MA.
15. Pollitt E, Gorman KS, Engle PL, Rivera JA, Martorell R. Nutrition in early life and the fulfillment of intellectual potential. J Nutr. 1995; 125(4 Suppl): $1111 \mathrm{~S}-1118 \mathrm{~S}$.

16. Hunter PR, Prüss-Ustün A. Have We Substantially Underestimated the Impact of Improved Sanitation Coverage on Child Health? A Generalized Additive Model Panel Analysis of Global Data on Child Mortality and Malnutrition. Ali M, editor. PLoS One. 2016;11(10): e0164571.

17. OMS. Estadisticas Sanitarias Mundiales 2015.2015.

18. NUD. Los objetivos de desarrollo sostenible y la iniciativa de ciudades prósperas.

19. Instituto Colombiano de Bienestar Familiar (ICBF). Resumen ejecutivo de la Encuesta Nacional de Situación Nutricional en Colombia (ENSIN). 2010.

20. OMS. Patrones internacionales de crecimiento infantil de la OMS. 2007; 42.

21. Long JS, Freese J. Regression Models For Categorical Dependent Variables Using Stata. A Stata Press Publ. 2001.

22. Benicio MHD, Martins APB, Venancio SI, Barros AJD de, Benício MHD, Martins APB, et al. Estimativas da prevalencia de desnutricao infantil nos municipios brasileiros em 2006. Rev Saude Publica. 2013; 47(3): 560-70.

23. Debnath A, Bhattacharjee N. Factors Associated with Malnutrition among Tribal Children in India: A Non-Parametric Approach. J Trop Pediatr. 2014; 60(3): 211-5.

24. Ortiz J, Van Camp J, Wijaya S, Donoso S, Huybregts L. Determinants of child malnutrition in rural and urban Ecuadorian highlands. Public Health Nutr. 2014; 17(09): 2122-2130.

25. Mwabu G. The Production of Child Health in Kenya: A Structural Model of Birth Weight. J Afr Econ. 2008; 18(2): 212-260.

26. Ewusie JE, Beyene J, Ahiadeke C, Hamid JS. Malnutrition in Pre-school Children across Different Geographic Areas and Socio-Demographic Groups in Ghana. Matern Child Health J. 2017; 21(4): 797-808.

27. Volpicelli K, Buttenheim AM. Do Social Factors Predict Appropriate Treatment of Child Diarrheal Disease in Peru? Matern Child Health J. 2016; 20(11): 2299-2308.

28. Man SL, Guo Y. Research on the social determinants of malnutrition among children under the age of 5 in China. Beijing Da Xue Xue Bao. 2016; 48(3): 418-423.

29. Bertranou FM, Delajara M, Amiune O. Una Función de Producción de Salud Infantil para Argentina. Universidad Nacional del Sur. 2002: 1-13.

30. Cuevas L, García G. La producción de salud infantil en Colombia: una aproximación. Desarrollo y Sociedad. 2007;59: 21-62. 